\title{
Cluster observations in the magnetosheath - Part 2: Intensity of the turbulence at electron scales
}

\author{
C. Lacombe ${ }^{1}$, A. A. Samsonov ${ }^{2}$, A. Mangeney ${ }^{1}$, M. Maksimovic $^{1}$, N. Cornilleau-Wehrlin ${ }^{3}$, C. C. Harvey ${ }^{4}$, \\ J.-M. Bosqued ${ }^{4}$, and P. Trávníćek ${ }^{5}$ \\ ${ }^{1}$ LESIA/CNRS, Observatoire de Paris, Meudon, France \\ ${ }^{2}$ Institute of Physics, St. Petersburg State University, St. Petersburg, Russia \\ ${ }^{3}$ Centre d'étude des Environnements Terrestre et Planétaire/UVSQ, Vélizy, France \\ ${ }^{4}$ Centre d'Etude Spatiale des Rayonnements/CNRS, Toulouse, France \\ ${ }^{5}$ Institute of Atmospheric Physics, Prague, Czech Republic
}

Received: 21 April 2006 - Revised: 23 October 2006 - Accepted: 10 November 2006 - Published: 21 December 2006

\begin{abstract}
The Cluster STAFF Spectral Analyser measures the magnetic and electric power spectral densities (PSD) $\delta B^{2}$ and $\delta E^{2}$ in the magnetosheath between $8 \mathrm{~Hz}$ and $4 \mathrm{kHz}$, i.e. between about the lower hybrid frequency and 10 times the proton plasma frequency. We study about $23 \mathrm{~h}$ of data on four different days. We do not consider the whistler waves and the electrostatic pulses (which are not always observed) but the underlying permanent fluctuations. Paper 1 (Mangeney et al., 2006) shows why the permanent PSD at a given frequency $f$ depends strongly on the angle $\Theta_{B V}$ between the magnetic field $\boldsymbol{B}$ and the flow velocity $\boldsymbol{V}$ : this is observed for the electromagnetic (e.m.) fluctuations, $\delta B^{2}$ and $\delta E_{e m}^{2}$, below the electron cyclotron frequency $f_{c e}$, and for the electrostatic (e.s.) fluctuations $\delta E_{e s}^{2}$ at and above $f_{c e}$. This dependence is due to the Doppler shift of fluctuations which have a highly anisotropic distribution of the intensity of the wave vector $\boldsymbol{k}$ spectrum, and have a power law intensity $\propto k^{-v}$ with $v \simeq 3$ to 4 . In the present paper, we look for parameters, other than $\Theta_{B V}$, which control the intensity of the fluctuations. At $f \simeq 10 \mathrm{~Hz}, \delta B^{2}$ and $\delta E_{e m}^{2}$ increase when the solar wind dynamic pressure $P_{D Y N}^{S W}$ increases. When $P_{D Y N}^{S W}$ increases, the magnetosheath $P_{D Y N}^{M S} \propto N V^{2}$ also increases, so that the local Doppler shift $(\boldsymbol{k} . \boldsymbol{V})$ increases for a given $\boldsymbol{k}$. If $\boldsymbol{V}$ increases, a given frequency $f$ will be reached by fluctuations with a smaller $k$, which are more intense: the variations of $\delta B^{2}(10 \mathrm{~Hz})$ with $P_{D Y N}^{S W}$ are only due to the Doppler shift in the spacecraft frame. We show that the e.m. spectrum in the plasma frame has an invariant shape $I_{1 D} \propto A_{e m}\left(k c / \omega_{p e}\right)^{-v}$ related to the electron inertial length $c / \omega_{p e}$ : the intensity $A_{e m}$ does not depend on $P_{D Y N}$, nor on the electron to proton temperature ratio $T_{e} / T_{p}$, nor on the upstream bow shock angle $\theta_{B N}$. Then, we show results of 3-D MHD numerical simulations of the magnetosheath plasma, which map the
\end{abstract}

Correspondence to: C. Lacombe

(Catherine.Lacombe@obspm.fr) regions where the angle $\Theta_{B V}$ is $\simeq 90^{\circ}$. The e.m. fluctuations are more intense in these magnetosheath regions, in the spacecraft frame where they are observed in the "whistler" range; and the e.s. fluctuations are less intense in these same regions, in the spacecraft frame where they are observed in the "ion acoustic" range. We conclude that the intensity of the permanent fluctuations in the e.m. range only depends on the Doppler shift, so that from day to day and from place to place in the magnetosheath, the $\boldsymbol{k}$ spectrum in the plasma frame has an invariant shape and a constant intensity. This is observed on scales ranging from $k c / \omega_{p e} \simeq 0.3(50 \mathrm{~km})$ to $k c / \omega_{p e} \simeq 30(500 \mathrm{~m})$, i.e. at electron scales smaller than the Cluster separation.

Keywords. Magnetospheric physics (Magnetosheath; Plasma waves and instabilities) - Space plasma physics (Turbulence)

\section{Introduction}

The STAFF Spectral Analyser on Cluster operates between $8 \mathrm{~Hz}$ and $4 \mathrm{kHz}$, i.e. between about the lower hybrid frequency and 10 times the proton plasma frequency $f_{p i}$ in the magnetosheath plasma. As discussed in the companion paper (Mangeney et al., 2006, Paper 1), two types of waves have to be distinguished in this frequency range: waves in the range $8 \mathrm{~Hz} \leq f \leq 300 \mathrm{~Hz}$, the "whistler" range, which are basically electromagnetic (e.m.), and waves in the range $300 \mathrm{~Hz} \leq f \leq 4 \mathrm{kHz}$, the "ion acoustic" range, which are basically electrostatic (e.s.). In Paper 1, we have shown that, for both types of waves, most of the variations of the power spectral density (PSD) observed at a given frequency $f$ can be explained by the variations of the Doppler shift $(\boldsymbol{k} . \boldsymbol{V})$, where $\boldsymbol{k}$ is the wave vector. In the "whistler" range, we have neglected the coherent whistler waves (which

Published by Copernicus GmbH on behalf of the European Geosciences Union. 
have a right-handed magnetic polarisation and are not always observed) and we have only considered the underlying permanent fluctuations. In this range, the magnetic and electric PSD increase strongly when the angle $\Theta_{B V}$ between the local magnetic field $\boldsymbol{B}$ and the plasma flow velocity $\boldsymbol{V}$ increases from $0^{\circ}$ to $90^{\circ}$. This can be modelled with statistically permanent e.m. fluctuations which have a negligible frequency in the plasma frame and are Doppler shifted up to $f$; the intensity of these fluctuations varies like a power law of the wave number $I_{1 D} \propto k^{-v}$ with $v \simeq 3$; the wave vectors of the fluctuations are mostly perpendicular to $\boldsymbol{B}$, i.e. with an intensity $I_{3 D} \propto\left|\sin \theta_{k B}\right|^{\mu} k^{-v-2}$ for $\mu \simeq 100$, in the wave number range $0.3<k c / \omega_{p e}<30 . \theta_{k B}$ is the angle between $\boldsymbol{k}$ and $\boldsymbol{B}$. Meanwhile, in the "ion acoustic" range, the e.s. PSD around $1 \mathrm{kHz}$ decreases when the angle $\Theta_{B V}$ increases up to $90^{\circ}$ : this can be modelled with statistically permanent e.s. fluctuations which have wave vectors mainly parallel to $\boldsymbol{B}$, i.e. with a 3-D intensity $I_{3 D} \propto\left|\cos \theta_{k B}\right|^{\mu} k^{-\nu-2}$, with $\mu \simeq 100$ and $v \simeq 4$, in the wave number range $0.1<k \lambda_{D e}<1$. These e.s. fluctuations may be ion acoustic modes Doppler shifted up to about $1 \mathrm{kHz}$. In this e.s. range (see Paper 1) we have not considered the electrostatic pulses: according to Pickett et al. (2005) the time duration and the amplitude of these pulses, observed in the time domain, do not depend on $\Theta_{B V}$.

Note that the above exponent $v$ refers to the power law index of the 1-D spectrum $I_{1 D}(k)$ defined by

$\delta B^{2}=\int d k I_{1 D}(k)$

with

$I_{1 D}(k)=2 \pi A k^{2} \int_{0}^{\pi} \sin \theta_{k B} d \theta_{k B} I_{3 D}\left(k, \theta_{k B}\right)$.

For an isotropic Kolmogorov spectrum $I_{1 D} \propto k^{-v}$ with $v=5 / 3$, the 3-D spectrum is $I_{3 D} \propto k^{-v-2}$.

Are there solar wind or magnetosheath parameters, other than $\Theta_{B V}$ i.e. the Doppler shift, which contribute to the control of the intensity of the fluctuations in the STAFF-SA frequency range? Indeed, at frequencies $f<10 \mathrm{~Hz}$, below the STAFF-SA range, several parameters have been found to have an effect upon the e.m. PSD in the magnetosheath: the solar wind Alfvén Mach number (Fairfield and Ness, 1970), the connection to quasi-parallel bow shocks (Luhmann et al., 1986; Czaykowska et al., 2001), and the magnetosheath proton $\beta_{p}$ (Anderson et al., 1994; Czaykowska et al., 2001). According to Rezeau et al. (1992) the level of the Alfvénic magnetic fluctuations close to the magnetopause is higher when the magnetopause moves earthward (compression) and lower when it moves outwards (expansion).

As for the e.m. waves above $10 \mathrm{~Hz}$, Rodriguez (1985) shows that their occurrence and their intensity mainly depend on the position in the magnetosheath: while this "whistler" turbulence is observed more frequently in the inner region of the sunward magnetosheath (a region where compressional
MHD fluctuations have been shown to dominate), the intensity of the $200 \mathrm{~Hz}$ whistler mode waves (broadband whistlers and narrowband lion roars) peaks near the magnetopause. For the e.s. waves around $1 \mathrm{kHz}$, Rodriguez (1979) finds that the wave intensity tends to maximize at the bow shock and to decrease towards the magnetopause and towards the dawn and dusk regions. In the "ion acoustic" range ( 0.2 to $4 \mathrm{kHz}$ ), the intensity of the waves observed in the dayside magnetosheath, just downstream of the bow shock ramp, does not appear to be correlated to the shock Alfvén Mach number, to the angle $\theta_{B N}$ between the upstream $\boldsymbol{B}$ field and the bow shock normal, or to the electron ratio $\beta_{e}$ (Onsager et al., 1989). In the distant magnetosheath, on ISEE-3, Coroniti et al. (1994) observed that the e.s. waves, between about $200 \mathrm{~Hz}$ and $3 \mathrm{kHz}$ ("ion acoustic" range), are less intense where the angle $\Theta_{B V}$ between the local magnetic field $\boldsymbol{B}$ and the plasma flow velocity $V$ reaches $90^{\circ}$. Between $8 \mathrm{~Hz}$ and $4 \mathrm{kHz}$, the wave intensity in the magnetosheath seems thus to be only controlled by the position in the magnetosheath and/or by the angle between $\boldsymbol{B}$ and $\boldsymbol{V}$.

We have seen in Paper 1 that the permanent PSD above $10 \mathrm{~Hz}$ in the magnetosheath was mainly controlled by $\Theta_{B V}$, i.e. by the Doppler shift. In the present paper, we show that the e.m. and the e.s. PSDs observed by STAFF-SA depend on the solar wind dynamic pressure $P_{D Y N}^{S W}$, but only at a given frequency in the spacecraft frame. At a given scale $k^{-1}$, normalised to the electron inertial length $c / \omega_{p e}$, the intensity of the e.m. $\boldsymbol{k}$ spectra in the plasma frame does not depend on $P_{D Y N}^{S W}$ (nor on $\theta_{B N}$ or $\beta_{p}$, see Sect. 3). As for the e.s. spectra, their intensity does not depend on $P_{D Y N}$ (nor on $\theta_{B N}$, $\beta_{p}$ or $T_{e} / T_{p}$, Sect. 4), if the frequency $f$ is normalised to the plasma frequency $f_{p i}$. The e.m. and e.s. turbulence intensities thus strongly depend on the Doppler shift through the angle $\Theta_{B V}$. Numerical 3-D MHD simulations (Sect. 5) allow us to map $\Theta_{B V}$ in the magnetosheath, as a function of the angle between the solar wind magnetic field $\boldsymbol{B}_{s w}$ and $\boldsymbol{X}_{\mathrm{GSE}}$ upstream of the bow shock. These maps of $\Theta_{B V}$ in the magnetosheath allow us to predict where the most intense "whistler" waves and the least intense "ion acoustic" waves will be found in the spacecraft frame.

\section{Data}

Every second, the STAFF-SA experiment (CornilleauWehrlin et al., 1997) measures the diagonal terms of the $5 \times 5$ spectral matrix, computed with the three components of the magnetic fluctuations $\delta \boldsymbol{B}$, and two components of the electric fluctuations $\delta \boldsymbol{E}$ (at 27 logarithmically spaced frequencies, between $8 \mathrm{~Hz}$ and $4 \mathrm{kHz}$ ). The variance of the fluctuations is nearly isotropic, so that $\delta B_{x x}^{2} \simeq \delta B_{y y}^{2} \simeq \delta B_{z z}^{2}$ and $\delta E_{x x}^{2} \simeq \delta E_{y y}^{2}$. We shall use 4-s averages of the total magnetic $\operatorname{PSD} \delta B^{2}=\delta B_{x x}^{2}+\delta B_{y y}^{2}+\delta B_{z z}^{2}$ in $\mathrm{nT}^{2} / \mathrm{Hz}$, and of the electric $\operatorname{PSD} \delta E^{2}=\delta E_{x x}^{2}+\delta E_{y y}^{2}$ in $(\mathrm{mV} / \mathrm{m})^{2} / \mathrm{Hz}$. 

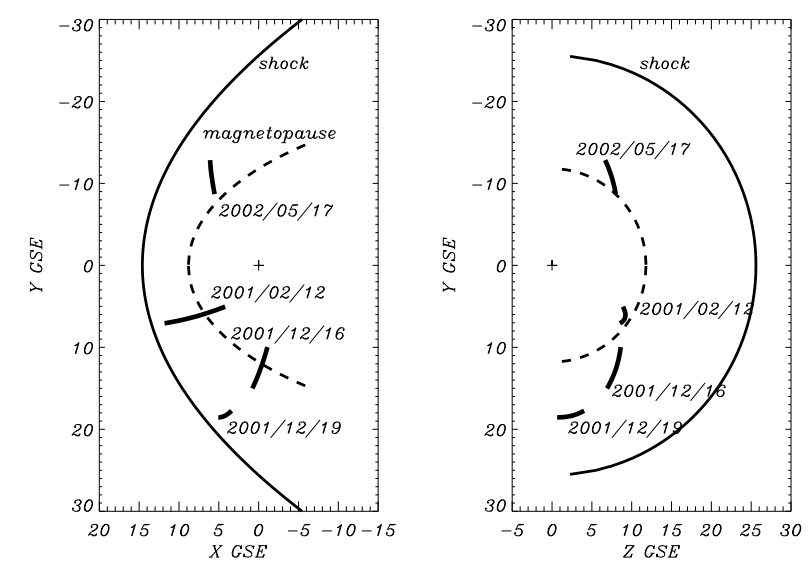

Fig. 1. For the four considered time intervals, projection of the Cluster orbit in the $\left(X_{\mathrm{GSE}}, Y_{\mathrm{GSE}}\right)$ and $\left(Z_{\mathrm{GSE}}, Y_{\mathrm{GSE}}\right)$ planes.

The data of Cluster 1 (Rumba), in the magnetosheath, are analysed during four intervals. Figure 1 displays the position of Cluster in the GSE planes (X, Y) and (Y, Z) for each interval. Also shown are a paraboloidal bow shock model without aberration, $X=14.6\left[1-\left(Y^{2}+Z^{2}\right) / 25.6^{2}\right]$ (Filbert and Kellogg, 1979), and a magnetopause model (dashed line; Sibeck et al., 1991), $Y^{2}+Z^{2}=139.2-0.18 X^{2}-14.2 X$. The considered intervals are generally far from the bow shock, except the longest interval, on 12 February 2001, which corresponds to a complete crossing of the magnetosheath. Cluster is in the dawn side on 17 May 2002. The plasma properties, proton density, temperature and velocity (Rème et al., 1997) and the electron temperature (Johnstone et al., 1997) are sampled with a time resolution of $4 \mathrm{~s}$. We use 4-s averages of the magnetic field (Balogh et al., 1997).

The date and the duration of the four intervals, lasting from $4 \mathrm{~h}$ to $7 \mathrm{~h}$, are given in the caption of Fig. 2, which displays relations between several plasma parameters. The different colours correspond to the four different days. Figure 2a shows the anticorrelations between $\beta_{p \|}=2 \mu_{0} N_{p} k_{B} T_{p \|} / B^{2}$ and $T_{p \perp} / T_{p \|}-1$, which are generally observed in the magnetosheath and which lead to the "bounded anisotropy model" (Denton et al., 1994, and references therein). $T_{p \|}$ and $T_{p \perp}$ are the proton temperatures, respectively, parallel and perpendicular to the $\boldsymbol{B}$ field. $P_{D Y N}^{M S}=m_{p} N_{p} V^{2}$ is the local dynamic pressure in the magnetosheath. Figure $2 \mathrm{~b}$ shows that it is correlated to $\beta_{p \|}$ for a given day. Figure $2 \mathrm{c}$ shows that when the angle $\Theta_{B V}$ increases towards $90^{\circ}, P_{D Y N}^{M S}$ generally increases for a given day. We shall see in Sect. 5 that this occurs in the magnetosheath flow when the solar wind (upstream) magnetic field and the $X_{\mathrm{GSE}}$ axis are quasiperpendicular. Figure $2 \mathrm{~d}$ displays an anticorrelation between $T_{e} / T_{p}$ and $P_{D Y N}^{M S}$, in the magnetosheath, which will be discussed in Sect. 6.
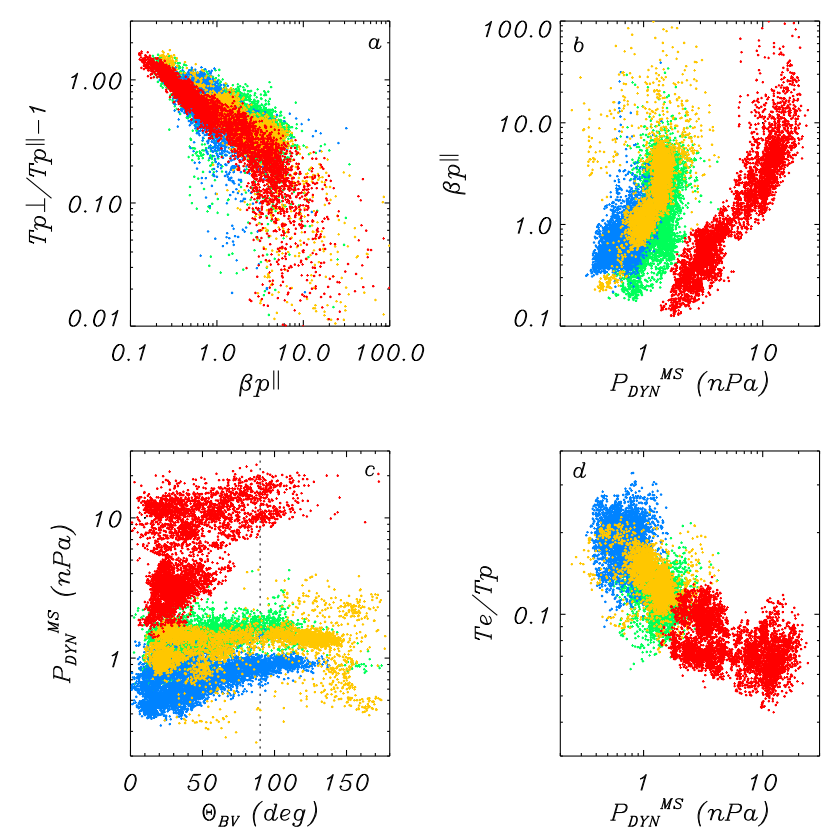

Fig. 2. On 12 February 2001 (00:15 to 07:30 UT, green points), 16 December 2001 (03:15 to 09:00 UT, red points), 19 December 2001 (00:00 to 06:00 UT, blue points), 17 May 2002 (08:00 to $12: 15$ UT, yellow points), some magnetosheath properties every $4 \mathrm{~s}$ : (a) the proton temperature anisotropy as a function of $\beta_{p \|}$, (b) $\beta_{p \|}$ as a function of $P_{D Y N}^{M S}=m_{p} N_{p} V^{2}$, (c) $P_{D Y N}^{M S}$ as a function of the angle $\Theta_{B V}$ between $\boldsymbol{B}$ and $\boldsymbol{V}$, (d) the electron to proton temperature ratio as a function of $P_{D Y N}^{M S}$.

\section{Intensity of the electromagnetic waves $(10$ to $100 \mathrm{~Hz})$}

\subsection{At a given frequency $\simeq 10 \mathrm{~Hz}$}

At the frequency $f=11 \mathrm{~Hz}$ (which is near the lower hybrid frequency), we look for a dependence of $\delta B^{2}$ on two solar wind parameters, the dynamic pressure $P_{D Y N}^{S W}$ and the bow shock angle $\theta_{B N}$. The solar wind data are those of ACE, near the Lagrange point L1. For each interval of magnetosheath data, we calculate the average time delay between ACE and Cluster which gives the best correlation between the magnetic field components on ACE and on Cluster. These delays vary between 57 and $74 \mathrm{mn}$, i.e. about $1 \mathrm{~h}$ for the four considered days. We thus know what are the upstream solar wind $\boldsymbol{B}$ field and $P_{D Y N}^{S W}$ at the time of the PSD measurements in the magnetosheath. The shock angle $\theta_{B N}$ between the upstream magnetic field and the shock normal is estimated with the assumption that the bow shock has the quoted paraboloidal shape and is located just upstream of the Cluster 1 position. We see in Fig. 3a that $\delta B^{2}$ increases when $P_{D Y N}^{S W}$ increases from day to day. There is no regular increase of $\delta B^{2}$ from day to day when the solar wind Mach number $M_{A}$ increases (not shown): $\delta B^{2}$ is related to $P_{D Y N}^{S W}$, not to $M_{A}$ which is 

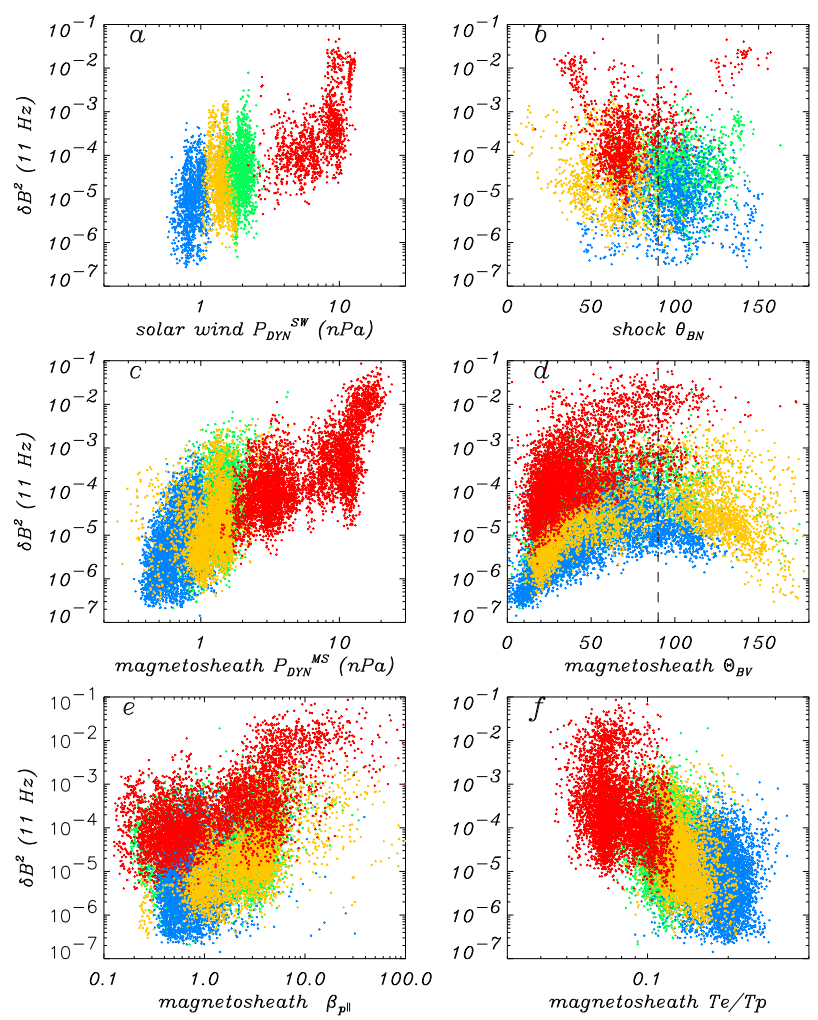

Fig. 3. The power spectral density (PSD) of the magnetic fluctuations at $11 \mathrm{~Hz}$, on the four considered days, as a function of: (a) the solar wind $P_{D Y N}^{S W}$ observed every $16 \mathrm{~s}$ on ACE, about $1 \mathrm{~h}$ earlier, (b) the upstream bow shock angle $\theta_{B N}$ every $16 \mathrm{~s}$, (c) the local magnetosheath $P_{D Y N}^{M S}$ every $4 \mathrm{~s}$, (d) the local angle $\Theta_{B V}$ between $\boldsymbol{B}$ and $\boldsymbol{V}$, (e) the local $\beta_{p \|}$, (f) the local ratio $T_{e} / T_{p}$.

proportional to $\left(P_{D Y N}^{S W}\right)^{1 / 2} / B$. Figure $3 \mathrm{~b}$ shows that the four intervals were mainly downstream of the quasi-perpendicular shocks; it seems that the waves are more intense for a few (red) points corresponding to $\theta_{B N}$ smaller than $45^{\circ}$ (quasiparallel shocks), but this is not confirmed on 19 December 2001 (blue points). We note in Fig. $3 \mathrm{c}$ that $\delta B^{2}(11 \mathrm{~Hz})$ is as well correlated to $P_{D Y N}^{M S}$ in the magnetosheath as it is correlated to $P_{D Y N}^{S W}$ (Fig. 3a). Indeed, there is a strong correlation between $P_{D Y N}^{S W}$ and $P_{D Y N}^{M S}$. We recall that the strong variations of $\delta B^{2}$ on a given day are due to the variation of the angle $\Theta_{B V}$ in the magnetosheath (see Fig. 3d, and Paper 1). As the intensity of the waves observed below $10 \mathrm{~Hz}$ in the magnetosheath is generally correlated to the local $\beta_{p \|}$ (see the Introduction), we look for this correlation at $11 \mathrm{~Hz}$. We see in Fig. $3 \mathrm{e}$ that there is a slight relation between $\delta B^{2}(11 \mathrm{~Hz})$ and $\beta_{p \|}$ on a given day, mainly on 16 December 2001 (red points). Finally, Fig. 3f shows how $\delta B^{2}(11 \mathrm{~Hz})$ decreases when the electron to proton temperature ratio, $T_{e} / T_{p}$, increases; this fact is related to the anticorrelation between $P_{D Y N}^{M S}$ and $T_{e} / T_{p}$ seen in Fig. $2 \mathrm{~d}$.
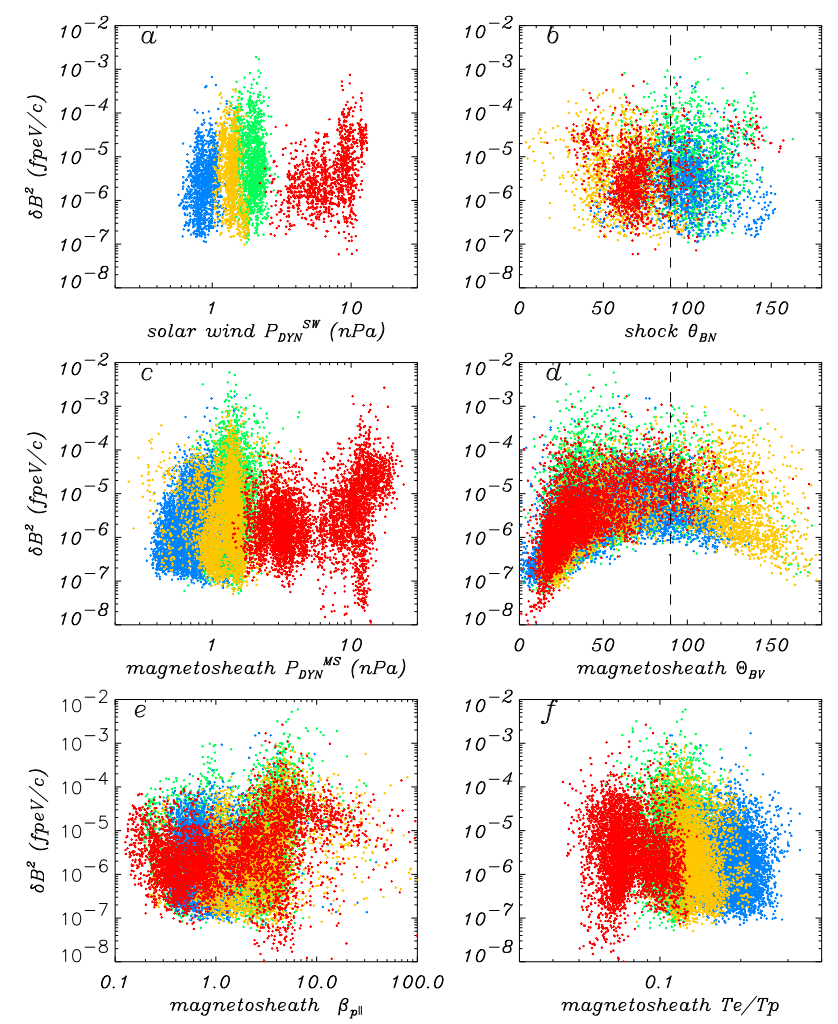

Fig. 4. The PSD $\delta B^{2}(f)$ interpolated at the time varying frequency $f_{p e} V / c$ on the four considered days, as a function of: (a) the solar wind $P_{D Y N}^{S W}$, (b) the upstream bow shock angle $\theta_{B N}$, (c) the magnetosheath $P_{D Y N}^{M S}$, (d) the angle $\Theta_{B V}$, (e) the magnetosheath $\beta_{p \|}$, (f) the ratio $T_{e} / T_{p}$.

\subsection{For a given scale $k c / \omega_{p e}=1$}

We have shown in Paper 1 that the observed frequencies $f$ of the e.m. waves only result from the Doppler shift of a spatial turbulence frozen in the plasma rest frame, with $\boldsymbol{k}$ mainly perpendicular to $\boldsymbol{B}$ in the range $k c / \omega_{p e} \simeq 0.3$ to 30 . Let us consider what happens to the scale $k^{-1}=c / \omega_{p e}$ if $\boldsymbol{k}$ is parallel to $\boldsymbol{V}$ : its Doppler shift $k V / 2 \pi$ will be $f_{D o p}=f_{p e} V / c$. As $f_{p e}$ and $\boldsymbol{V}$ vary on each day and from day to day, the frequency $f_{\text {Dop }}$ at which the considered scale is observed will vary like $V N_{p}^{1 / 2}$, which itself varies like the square root of the local $P_{D Y N}$. In Fig. 4, we display $\delta B^{2}(f)$ interpolated at the time dependent frequency $f_{p e} V / c$. Figures $4 a$, $4 \mathrm{c}$ and $4 \mathrm{f}$ show that $\delta B^{2}\left(f_{p e} V / c\right)$ does not depend anymore on $P_{D Y N}^{S W}$, on $P_{D Y N}^{M S}$ and on $T_{e} / T_{p}$. There is strictly no dependence of the PSD on the shock angle $\theta_{B N}$ (Fig. 4b) and on $\beta_{p \|}$ (Fig. 4e), even on 16 December 2001 (red points). The only variation is with $\Theta_{B V}$ (Fig. 4d). This regular variation, also observed at a given frequency (Fig. 3d), is due to the fact that the intense fluctuations are those with a small $k$ and with $\boldsymbol{k}$ mostly perpendicular to $\boldsymbol{B}$ (see Paper 1). Indeed, 
a given Doppler shift $f_{D o p}=k V \cos \theta_{k V} / 2 \pi$ will be reached by a small $k$ (with a large intensity), if $\theta_{k V}=0^{\circ}$ : this happens for $\Theta_{B V}=90^{\circ}$; and $f_{D o p}$ will be reached by a large $k$ (small intensity) for $\Theta_{B V}=0^{\circ}$.

Besides $c / \omega_{p e}$, we consider another electron scale, the electron gyroradius $r_{g e}$ : we then have $f_{D o p}=f_{c e} V / v_{\text {the }}$ for the scale $k^{-1}=r_{g e}$, if $\boldsymbol{k}$ is parallel to $\boldsymbol{V}$. The scatter plots of $\delta B^{2}\left(f_{c e} V / v_{\text {the }}\right)$ as a function of $P_{D Y N}^{M S}$ or of $\Theta_{B V}$ (not shown) are more dispersed than the scatter plots of Figs. $4 \mathrm{c}$ and $4 \mathrm{~d}$. The scale of the turbulence in the e.m. range is thus $c / \omega_{p e}, \operatorname{not} r_{g e}$.

We conclude that the variations of the e.m. PSD $\delta B^{2}$ at a given frequency are mainly due to the variations of the local Doppler shift $\propto V N_{p}^{1 / 2}$ for wavelengths normalised to the scale $c / \omega_{p e}$. This implies that the 3-D spectrum $\delta B^{2}(\boldsymbol{k}) \propto A_{e m}\left(k c / \omega_{p e}\right)^{-\nu-2}\left|\sin \theta_{k B}\right|^{\mu}$ in the plasma frame is broadly invariant on a given day, and from day to day, i.e. with a value of $A_{e m}$ constant when $P_{D Y N}^{S W}$ and $P_{D Y N}^{M S}$ varies.

\section{Intensity of the electrostatic waves $(\simeq 1 \mathrm{kHz})$}

We have shown in Paper 1 that the magnetic fluctuations $\delta B^{2}$ for $k c / \omega_{p e} \simeq 0.3$ to 30 have wave vectors mainly perpendicular to $\boldsymbol{B}$; the e.m. electric fluctuations $\delta E_{e m}^{2}$, at the same frequencies, have similar properties. For smaller scales, $k c / \omega_{p e} \simeq 15$ to $150\left(k \lambda_{D e} \simeq 0.1\right.$ to 1$)$, the electrostatic fluctuations $\delta E_{e s}^{2}$ have $\boldsymbol{k}$ mainly parallel to $\boldsymbol{B}$. To account for the variations of $\delta E^{2}$ with $f$ and $\Theta_{B V}$, we have been led to assume that, at $k c / \omega_{p e} \simeq 30, \delta E_{e s}^{2} \simeq 300 \delta E_{e m}^{2}$ (Paper 1).

We look for a dependence of $\delta E_{e s}^{2}$ on the magnetosheath dynamic pressure $P_{D Y N}^{M S}$, itself correlated to the solar wind $P_{D Y N}^{S W}$. We only consider the data when $\Theta_{B V}$ is large $\left(70^{\circ}\right.$ to $110^{\circ}$ ), because the dispersion of $\delta E_{e s}^{2}$ at a given $f$ is weaker when $\Theta_{B V}$ is large (see Fig. 5e of Paper 1). Figures 5a and $5 \mathrm{~b}$ display $\delta E^{2}$ at a given frequency $1414 \mathrm{~Hz}$ as a function of $P_{D Y N}^{M S}$ and of $T_{e} / T_{p}$. The broken black line gives the median values in bins of $T_{e} / T_{p}$ with a width equal to 0.02 . $\delta E^{2}$ tends to increase when $P_{D Y N}^{M S}$ increases from day to day. As $P_{D Y N}^{M S}$ is anticorrelated with $T_{e} / T_{p}$ (Fig. $\left.2 \mathrm{~d}\right), \delta E^{2}(1414 \mathrm{~Hz})$ decreases when $T_{e} / T_{p}$ increases (Fig. 5b). There is no correlation between $\delta E^{2}(1414 \mathrm{~Hz})$ and the bow shock angle $\theta_{B N}$ or the magnetosheath $\beta_{p \|}$ (not shown).

Then, we interpolate $\delta E^{2}(f)$ at a time-dependent frequency, 1.5 times the proton plasma frequency $f_{p i}$. In Figs. $5 \mathrm{c}$ and $5 \mathrm{~d}$ we note that $\delta E^{2}\left(1.5 f_{p i}\right)$ does not depend anymore on $P_{D Y N}^{M S}$ and $T_{e} / T_{p}$. It does not depend on $\beta_{p \|}$ nor on the shock angle $\theta_{B N}$ (not shown). The spectrum of the e.s. fluctuations is thus invariant with respect to an energy input like $P_{D Y N}^{S W}$, if the frequency is normalised to the local plasma frequency $f_{p i}$.
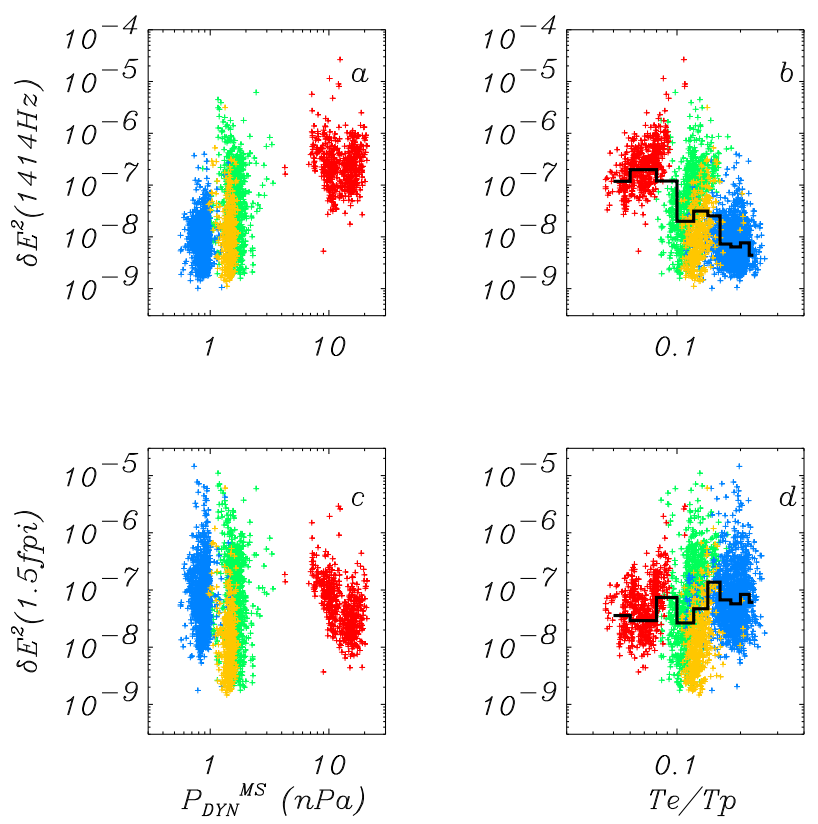

Fig. 5. For the time intervals when $\Theta_{B V}$ in the magnetosheath is between $70^{\circ}$ and $110^{\circ}$, (a) and (b) the e.s. PSD $\delta E^{2}$ at $1414 \mathrm{~Hz}$ as a function of $P_{D Y N}^{M S}$ and $T_{e} / T_{p}$, (c) and (d) the e.s. PSD $\delta E^{2}(f)$, interpolated at the time varying frequency $1.5 f_{p i}$, as a function of $P_{D Y N}^{M S}$ and $T_{e} / T_{p}$. The broken black lines give the median values in bins of $T_{e} / T_{p}$ with a width 0.02 .

\section{Wave intensity and position in the magnetosheath}

Paper 1, and the Sect. 3 of the present paper led us to conclude that the intensities of the e.m. and e.s. fluctuations at a given frequency in the magnetosheath strongly depend on the local Doppler shift. They will depend on the position in the magnetosheath (as observed by Rodriguez, 1979, 1985), if the Doppler shift itself, i.e. the flow speed $\boldsymbol{V}$ and the angle $\Theta_{B V}$, depend on the position.

To map the regions of strong Doppler shift, 3-D MHD simulations of the magnetosheath plasma have been performed (see Samsonov and Hubert, 2004; Samsonov, 2006). Figure 6 gives the flow and field parameters calculated in the middle of the magnetosheath (i.e. on a surface at equal distance from the shock and the magnetopause) and projected in the $\left(Y_{B}, Z_{B}\right)$ plane, which is the $\left(Y_{\mathrm{GSE}}, Z_{\mathrm{GSE}}\right)$ plane rotated around the $X_{\mathrm{GSE}}$ axis, so that the direction of $Y_{B}$ is parallel to the projection of the upstream solar wind magnetic field $\boldsymbol{B}_{s w}$ in the $\left(Y_{\mathrm{GSE}}, Z_{\mathrm{GSE}}\right)$ plane. This projection is the red arrow in Fig. 6c, with $B_{X s w} \leq 0, B_{Y s w}>0$ and $B_{Z s w}=0$. The mapped values, density, speed and magnetic field intensity, are normalised to the solar wind values. Figures $6 \mathrm{a}-\mathrm{d}$ are drawn for a cone angle of the solar wind magnetic field $\left(\boldsymbol{B}_{s w}, \boldsymbol{X}_{\mathrm{GSE}}\right)=45^{\circ}$. The density distribution in the magnetosheath is nearly axisymmetric (Fig. 6a). The velocity 

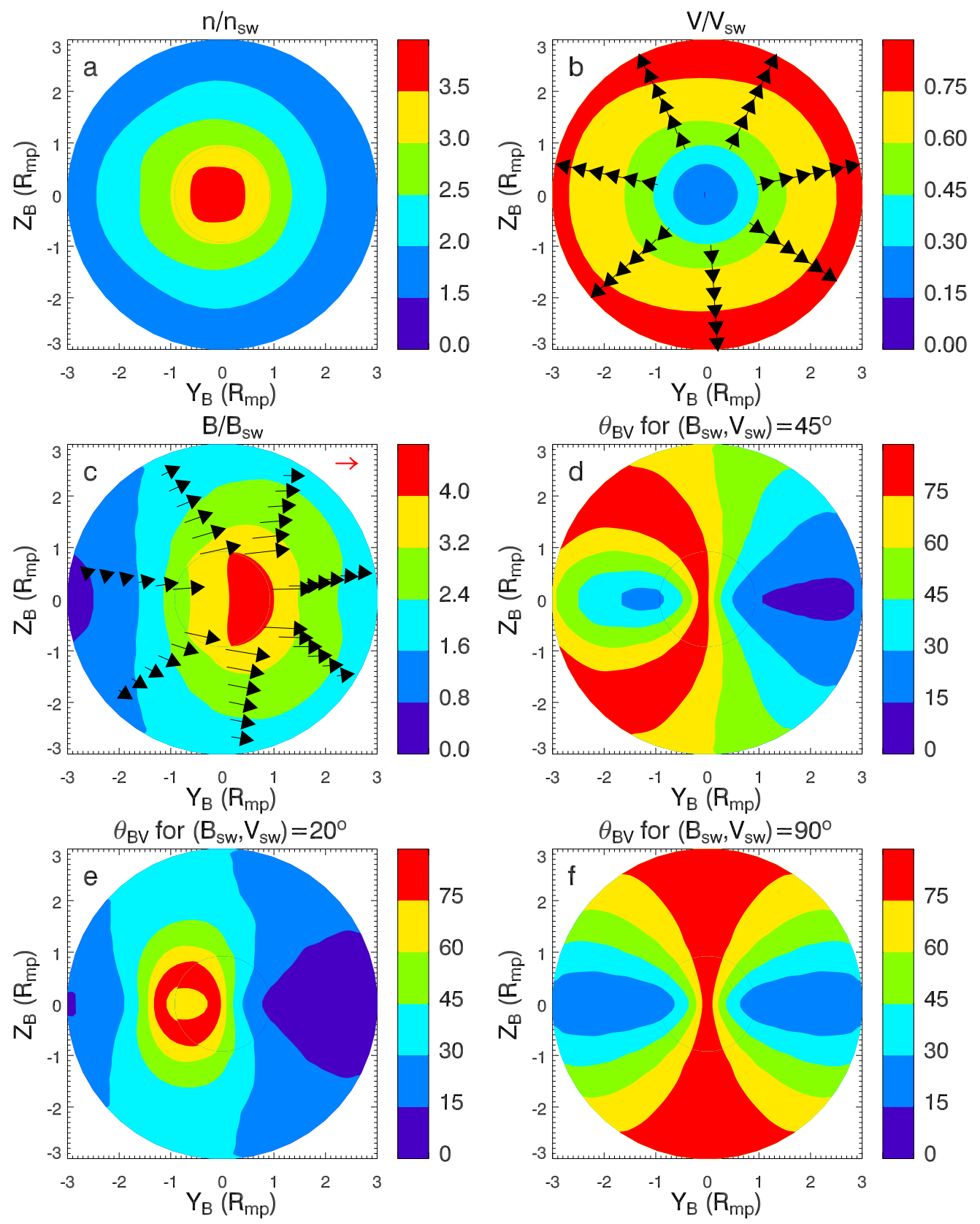

Fig. 6. Results of 3-D MHD simulations. The flow and field values calculated in the middle of the magnetosheath are projected in the $\left(Y_{B}, Z_{B}\right)$ plane. This plane is the $\left(Y_{\mathrm{GSE}}, Z_{\mathrm{GSE}}\right)$ plane rotated around $X_{\mathrm{GSE}}$, so that the direction $Y_{B}$ is parallel to the projection of the solar wind magnetic field $\boldsymbol{B}_{s w}$ in the $\left(Y_{\mathrm{GSE}}, Z_{\mathrm{GSE}}\right)$ plane. $R_{m p}$ is about $11 R_{E}$. For a solar wind cone angle $\left(\boldsymbol{B}_{s w}, \boldsymbol{X}_{\mathrm{GSE}}\right)=45^{\circ}:(\mathbf{a})$ the plasma density normalised to the solar wind density, (b) the velocity normalised to $V_{s w}$; the arrows give the direction of $\boldsymbol{V}$, (c) the magnetic field normalised to $B_{S w}$; the arrows give the direction of $\boldsymbol{B},(\mathbf{d})$ the angle $\Theta_{B V},(\mathbf{e}) \Theta_{B V}$ for the solar wind cone angle $\left(\boldsymbol{B}_{s w}, \boldsymbol{X}_{\mathrm{GSE}}\right)=20^{\circ},(\mathbf{f})$ $\Theta_{B V}$ for $\left(\boldsymbol{B}_{s w}, \boldsymbol{X}_{\mathrm{GSE}}\right)=90^{\circ}$.

directions projected in the $\left(Y_{B}, Z_{B}\right)$ plane (black arrows in Fig. $6 \mathrm{~b}$ ) are not exactly radial, and the largest values of the modulus of $\boldsymbol{V}$ are found at high latitudes in the $\left(Y_{B}, Z_{B}\right)$ plane. The directions of the projected $\boldsymbol{B}$ field (black arrows in Fig. 6c) make small angles with the $Y_{B}$ direction (i.e. with the projection of $\boldsymbol{B}_{s w}$ ): the clock angle of the $\boldsymbol{B}$ field remains nearly constant through the bow shock. The angle $\Theta_{B V}$ is displayed in Fig. 6d: the red regions are the regions where $\Theta_{B V}$ is large, between $75^{\circ}$ and $90^{\circ}$. Except in the subsolar regions $\left(\left(Y_{B}^{2}+Z_{B}^{2}\right)^{1 / 2} \leq 0.5 R_{m p}\right.$, where Cluster does not 
cruise) the value of $\Theta_{B V}$ mainly depends on the latitude in the plane $\left(Y_{B}, Z_{B}\right)$. Figure 6e gives the angle $\Theta_{B V}$ when the cone angle of the solar wind $\boldsymbol{B}$ field is $\left(\boldsymbol{B}_{s w}, \boldsymbol{X}_{\mathrm{GSE}}\right)=20^{\circ}$ : the largest values $\Theta_{B V}>75^{\circ}$ are now at low latitudes, for $Y_{B}<0$, downstream of a quasi-parallel bow shock, and the smallest $\Theta_{B V}$ are also at low latitudes, downstream of a quasiperpendicular bow shock $\left(Y_{B}>0\right)$. If the angle $\left(\boldsymbol{B}_{s w}, \boldsymbol{X}_{\mathrm{GSE}}\right)$ is $90^{\circ}$ (Fig. 6f), $\Theta_{B V}$ is larger at high latitudes in the plane $\left(Y_{B}, Z_{B}\right)$. A comparison of Fig. 6b with Fig. $6 \mathrm{f}$ shows that $V$ tends to be larger when $\Theta_{B V}$ is large. This is typical of a magnetosheath observed when $\left(B_{s w}, X_{\mathrm{GSE}}\right)$ is larger than $45^{\circ}$, and implies that the dynamic pressure will increase when $\Theta_{B V}$ increases (see Fig. 2c).

These simulations show that the angle $\Theta_{B V}$ will be larger for large $\left|Z_{B}\right|$ values if the cone angle of the solar wind magnetic field is between $45^{\circ}$ and $90^{\circ}$. On 17 May 2002, the observed cone angle $\left(\boldsymbol{B}_{s w}, \boldsymbol{X}_{\mathrm{GSE}}\right)$ remains between $45^{\circ}$ and $90^{\circ}$, while Cluster remains nearly in the same GSE position. Yet, as the magnetic field rotates, the direction $Y_{B}$ rotates, and the position of Cluster follows large arcs of circle in the $\left(Y_{B}, Z_{B}\right)$ plane of Fig. 7. Figure 7a gives the position of Cluster in the $\left(Y_{B}, Z_{B}\right)$ frame when the e.m. PSD $\delta B^{2}$ at $11 \mathrm{~Hz}$ is large, precisely for $10 \%$ of the most intense values of $\delta B^{2}$ over 4 h. Similarly, Fig. $7 \mathrm{~b}$ gives the position of Cluster when the e.s. PSD $\delta E^{2}$ at $1414 \mathrm{~Hz}$ is large. We see that the most intense e.m. fluctuations are found at large $Z_{B}$, while the most intense e.s. fluctuations are found at small $Z_{B}$.

We conclude that the Doppler shift (the angle $\Theta_{B V}$ and the flow speed) depends on the position in the magnetosheath for a given direction of the solar wind magnetic field $\boldsymbol{B}_{s w}$; and it depends strongly on the direction of $\boldsymbol{B}_{s w}$.

\section{Discussion}

We have shown in Sect. 3 that the correlation between the magnetosheath dynamic pressure, $P_{D Y N}^{M S}$ and $\delta B^{2}$, at a given frequency $(11 \mathrm{~Hz})$ is only due to the Doppler shift, itself proportional to $P_{D Y N}^{M S}$. This correlation between $\delta B^{2}(11 \mathrm{~Hz})$ and $P_{D Y N}^{M S}$ implies some correlations between $\delta B^{2}(11 \mathrm{~Hz})$ and $\beta_{p \|}$, because $\beta_{p \|}$ is correlated with $P_{D Y N}^{M S}$, at least for a given day (Fig. 2c; see also Farrugia et al., 2000). Some observations display a correlation between $\delta B^{2}$ below $10 \mathrm{~Hz}$ (ULF waves) and $\beta_{p \|}$. This correlation in the spacecraft frame is questionable because it could be partly due to the Doppler shift, not to a role played by $\beta_{p \|}$.

We have seen in Sect. 3 that there is no significant relation between the e.m. wave intensity and the bow shock angle $\theta_{B N}$, neither at a given frequency (Fig. 3b) nor for a given scale (Fig. 4b). In fact, our magnetosheath intervals generally correspond to quasi-perpendicular shocks, so that we cannot check whether the fluctuations are more intense downstream of quasi-parallel shocks. However, the numerical simulations of Figs. $6 \mathrm{~d}, 6 \mathrm{e}$ and $6 \mathrm{f}$ show that the value of the angle $\Theta_{B V}$ at a given place in the $\left(Y_{B}, Z_{B}\right)$ plane depends strongly on the
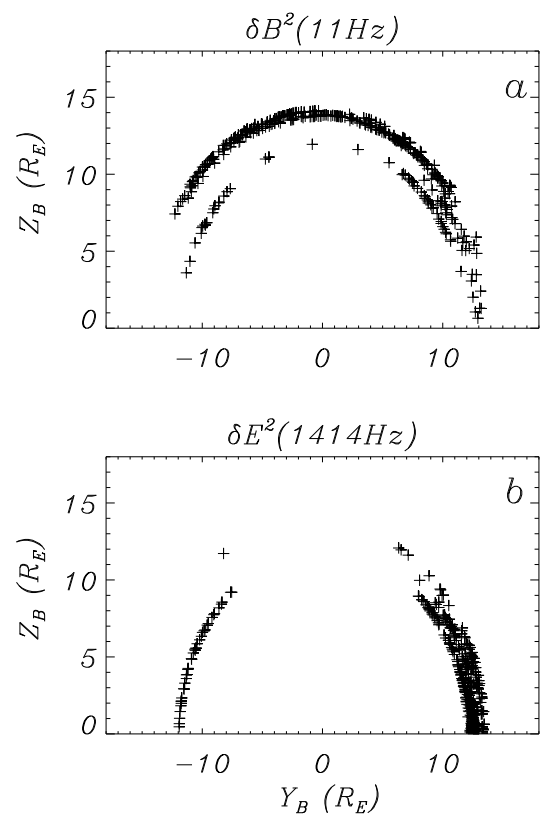

Fig. 7. The plane $\left(Y_{B}, Z_{B}\right)$ is the $\left(Y_{\mathrm{GSE}}, Z_{\mathrm{GSE}}\right)$ plane rotated around $X_{\mathrm{GSE}}$, so that the direction $Y_{B}$ is parallel to the projection of the local magnetosheath $\boldsymbol{B}$ field in the $\left(Y_{\mathrm{GSE}}, Z_{\mathrm{GSE}}\right)$ plane. Wave data on 17 May 2002, 08:00 to 12:15 UT: (a) the position of Cluster in the $\left(Y_{B}, Z_{B}\right)$ plane for $10 \%$ of the most intense PSD $\delta B^{2}$ at $11 \mathrm{~Hz}$ (electromagnetic fluctuations), (b) the position of Cluster in the $\left(Y_{B}, Z_{B}\right)$ plane for $10 \%$ of the most intense PSD $\delta E^{2}$ at $1414 \mathrm{~Hz}$ (electrostatic fluctuations).

cone angle $\left(\boldsymbol{B}_{s w}, \boldsymbol{X}_{\mathrm{GSE}}\right)$, and thus on the bow shock angle $\theta_{B N}$. Thus, a possible dependence of $\delta B^{2}$ on $\theta_{B N}$ could be due to the dependence on the angle $\Theta_{B V}$ which is simply on the Doppler shift for anisotropic $\boldsymbol{k}$ spectra.

We mentioned in Sect. 2 that there is an anticorrelation between $T_{e} / T_{p}$ and $P_{D Y N}^{M S}$ in the magnetosheath for a given day and from day to day (Fig. 2d): for the four days (21 000 points) the correlation coefficient between $P_{D Y N}^{M S}$ and $T_{e} / T_{p}$ is -0.82 (the correlation is 0.77 between $P_{D Y N}^{M S}$ and $T_{p}$, and -0.66 between $P_{D Y N}^{M S}$ and $T_{e}$ ). In the solar wind, it is well known that $T_{p}$ is larger than $T_{e}$ in the high-speed wind, and $T_{e}$ larger than $T_{p}$ in the low-speed wind. There is a global anticorrelation between $T_{e} / T_{p}$ and $V_{s w}$ (see Mangeney et al., 1999). This anticorrelation upstream of the bow shock will probably also be observed downstream of the bow shock. Indeed, a subcritical shock tends to heat the electrons, while a supercritical shock, with a higher Mach number (i.e. a higher flow speed), tends to heat the protons more than the electrons. The shock will strengthen the anticorrelation between $T_{e} / T_{p}$ and $\boldsymbol{V}$, and strengthen the anticorrelation between $T_{e} / T_{p}$ and $P_{D Y N}^{M S}$ in the magnetosheath. We thus suggest that this latter anticorrelation comes from a solar wind property amplified by the shock heating. 


\section{Conclusions}

In this paper, we have not considered the fluctuations like the e.m. whistler or the e.s. solitary waves which are intermittently observed in the magnetosheath, but only the underlying permanent fluctuations, between about the nominal lower hybrid frequency and 10 times the nominal proton plasma frequency.

In the magnetosheath, the permanent electromagnetic power spectral density, observed at a given frequency in the spacecraft frame, increases when the solar wind and the magnetosheath dynamic pressure $P_{D Y N}^{S W}$ and $P_{D Y N}^{M S}$ increase. This correlation is a consequence of the Doppler shift, and disappears if we consider the fluctuations at a given scale $\left(\propto k^{-1}\right)$, not at a given frequency. Indeed, we find that the scale of the electromagnetic fluctuations is related to the electron inertial length $c / \omega_{p e}$ : the e.m. PSD has a $k$ spectral intensity $I_{1 D} \propto\left(k c / \omega_{p e}\right)^{-v}$ with $v \simeq 3$ to 4 , for scales ranging from $k c / \omega_{p e} \simeq 0.3$ to 30 . The directions of the wave vectors $\boldsymbol{k}$ are mostly perpendicular to the $\boldsymbol{B}$ field (see Paper 1), so that the e.m. 3-D $\boldsymbol{k}$ spectrum (assumed axisymmetric with respect to $\boldsymbol{B})$ varies like $A_{e m}\left(k c / \omega_{p e}\right)^{-\nu-2}\left|\sin \theta_{k B}\right|^{\mu}$ with $\mu \simeq 100$. We find that $A_{e m}$ is invariant, it does not depend on $P_{D Y N}^{S W}$ or $P_{D Y N}^{M S}$, nor on the magnetosheath $\beta_{p \|} \cdot A_{e m}$ does not depend anymore on the upstream bow shock angle $\theta_{B N}$ (but our observations correspond to a limited range of $\theta_{B N}$, downstream of oblique or quasi-perpendicular bow shocks).

The electrostatic permanent fluctuations are probably Doppler shifted ion acoustic waves for scales ranging from $k \lambda_{D e} \simeq 0.1$ to 1 . The directions of $\boldsymbol{k}$ are mostly parallel to the $\boldsymbol{B}$ field (see Paper 1).

A consequence of the strong anisotropies of the e.m. and e.s. $\boldsymbol{k}$ spectra is that the e.m. fluctuations are more intense and the e.s. fluctuations less intense, at a given frequency in the spacecraft frame, when the local angle $\Theta_{B V}$ between the flow velocity and the $\boldsymbol{B}$ field is close to $90^{\circ}$. 3-D MHD simulations of the flow in the magnetosheath allowed us to map the regions where $\Theta_{B V}$ reaches $90^{\circ}$. These maps depend strongly on the cone angle $\left(\boldsymbol{B}_{s w}, \boldsymbol{X}_{\mathrm{GSE}}\right)$ of the solar wind magnetic field. For $\left(\boldsymbol{B}_{s w}, \boldsymbol{X}_{\mathrm{GSE}}\right)$ larger than $45^{\circ}$, the angle $\Theta_{B V}$ reaches $90^{\circ}$ in regions with a high latitude with respect to the $\left(X_{\mathrm{GSE}}, B\right)$ plane; $\boldsymbol{B}$ is the direction of the magnetosheath magnetic field, which remains in a plane nearly parallel to the solar wind $\left(X_{\mathrm{GSE}}, B_{s w}\right)$ plane. Then, the e.m. PSD will be more intense at high latitudes, and the e.s. PSD more intense at low latitudes with respect to the $\left(X_{\mathrm{GSE}}, B\right)$ plane. For a cone angle of the solar wind magnetic field smaller than $45^{\circ}$, the angle $\Theta_{B V}$ reaches $90^{\circ}$ downstream of the quasi-parallel bow shock, at low latitudes with respect to the $\left(X_{\mathrm{GSE}}, B\right)$ plane: the e.m. PSD will be more intense there, and the e.s. PSD less intense.

We conclude that the intensity (in the spacecraft frame) of the e.m. and e.s. fluctuations in the magnetosheath strongly depend on the Doppler effect. This is observed on scales ranging from $k c / \omega_{p e} \simeq 0.3(50 \mathrm{~km})$ to $k \lambda_{D e} \simeq 1(30 \mathrm{~m})$, i.e. at electron scales, smaller than the Cluster separation. The Doppler shift is related to the dynamic pressure $P_{D Y N}$ and to the angle $\Theta_{B V}$; the angle $\Theta_{B V}$ is itself related to the shock angle $\theta_{B N}$ and to the position in the magnetosheath. Thus, all the parameters $\left(P_{D Y N}, \theta_{B N}\right.$, position in the magnetosheath) which have been shown to influence the wave intensity, from the ULF range to the "whistler" and "ion acoustic" ranges, may partly be manifestations of the Doppler effect.

Acknowledgements. We are very grateful to the team of the Cluster Magnetic field investigation (PIs A. Balogh and E. Lucek). We thank A. Fazakerley for the use of the Peace data. We thank the ACE MAG and SWEPAM instrument teams and the ACE Science Center for providing the ACE data. It is a pleasure to thank Jolene Pickett for useful discussions, and O. Santolìk for the program PRASSADCO. We thank the three referees for useful comments.

Topical Editor I. A. Daglis thanks three referees for their help in evaluating this paper.

\section{References}

Anderson, B. J., Fuselier, S. A., Gary, S. P., and Denton, R. E.: Magnetic spectral signatures in the Earth's magnetosheath and plasma depletion layer, J. Geophys.Res., 99, 5877-5891, 1994.

Balogh, A., Dunlop, M. W., Cowley, S. W. H., Southwood, D. J., Thomlinson, J. G., Glassmeier, K. H., Musmann, G., Lühr, H., Buchert, S., Acuña, M. H., Fairfield, D. H., Slavin, J. A., Riedler, W., Schwingenschuh, K., Kivelson, M. G., and the Cluster magnetometer team: The Cluster Magnetic Field Investigation, Space Science Reviews, 79, 65-92, 1997.

Cornilleau-Wehrlin, N., Chauveau, P., Louis, S., Meyer, A., Nappa, J. M., Perraut, S., Rezeau, L., Robert, P., Roux, A., de Villedary, C., de Conchy, Y., Friel, L., Harvey, C. C., Hubert, D., Lacombe, C., Manning, R., Wouters, F., Lefeuvre, F., Parrot, M., Pinçon, J.L., Poirier, B., Kofman, W., Louarn, P., and the Staff investigator team: The Cluster Spatio-temporal Analysis of Field Fluctuations (Staff) Experiment, Space Science Reviews, 79, 107-136, 1997.

Coroniti, F. V., Greenstadt, E. W., Moses, S. L., Tsurutani, B. T., and Smith, E. J.: On the absence of plasma wave emissions and the magnetic field orientation in the distant magnetosheath, Geophys. Res. Lett, 21, 2761-2764, 1994.

Czaykowska, A., Bauer, T. M., Treumann, R. A., and Baumjohann, W.: Magnetic field fluctuations across the Earth's bow shock, Ann. Geophys., 19, 275-287, 2001, http://www.ann-geophys.net/19/275/2001/.

Denton, R. E., Anderson, B. J., Gary, S. P., and Fuselier, S. A.: Bounded anisotropy fluid model for ion temperatures, J. Geophys. Res., 99, $11225-11241,1994$.

Fairfield, D. H. and Ness, N. F.: Magnetic field fluctuations in the Earth's magnetosheath, J. Geophys. Res., 75, 6050-6060, 1970.

Farrugia, C. J., Erkaev, N. V., and Biernat, H. K.: On the effect of solar wind dynamic pressure on the anisotropic terrestrial magnetosheath, J. Geophys. Res., 105, 115-127, 2000.

Filbert, P. C. and Kellogg, P. J.: Electrostatic noise at the plasma frequency beyond the Earth's bow shock, J. Geophys. Res., 84, 1369-1381, 1979. 
Johnstone, A. D., Alsop, C., Burge, S., Carter, P. J., Coates, A. J., Coker, A. J., Fazakerley, A. N., Grande, M. Gowen, R. A., Gurgiolo, C., Hancock, B. K., Narheim, B., Preece, A., Sheather, P. H., Winningham, J. D., and Woodliffe, R. D.: Peace: a Plasma Electron and Current Experiment, Space Science Reviews, 79, 351-398, 1997.

Luhmann, J. G., Russell, C. T., and Elphic, R.C.: Spatial distribution of magnetic field fluctuations in the dayside magnetosheath, J. Geophys. Res., 91, 1711-1715, 1986.

Mangeney, A., Salem, C., Lacombe, C., Bougeret, J.-L., Perche, C., Manning, R., Kellogg, P. J., Goetz, K., Monson, S. J., and Bosqued, J.-M.: WIND observations of coherent electrostatic waves in the solar wind, Ann. Geophys., 17, 307-320, 1999, http://www.ann-geophys.net/17/307/1999/.

Mangeney, A., Lacombe, C., Maksimovic, M., Samsonov, A. A., Cornilleau-Wehrlin, N., Harvey, C. C., Bosqued, J.-M., and Trávníček, P.: Cluster observations in the magnetosheath - Part 1: Anisotropies of the wave vector distribution of the turbulence at electron scales, Ann. Geophys., 24, 3507-3521, 2006, http://www.ann-geophys.net/24/3507/2006/.

Onsager, T. G., Holzworth, R. H., Koons, H. C., Bauer, O. H., Gurnett, D. A., Anderson, R. R., Lühr, H., and Carlson, C. W.: Highfrequency electrostatic waves near the Earth's bow shock, J. Geophys. Res., 94, 13 397-13 408, 1989.

Pickett, J. S., Chen, L.-J., Kahler, S. W., Santolik, O., Goldstein, M. L., Lavraud, B., Décréau, P. M. E., Kessel, R., Lucek, E., Lakhina, G. S., Tsurutani, B. T., Gurnett, D. A., CornilleauWehrlin, N., Fazakerley, A., Rème, H., and Balogh, A.: On the generation of solitary waves observed by Cluster in the nearEarth magnetosheath, Nonlin. Processes Geophys., 12, 181-193, 2005 ,

http://www.nonlin-processes-geophys.net/12/181/2005/.
Rème, H., Bosqued, J.-M., Sauvaud, J. A., Cros, A., Dandouras, J., Aoustin, C., Bouyssou, J., Camus, T., Cuvilo, J., Martz, C., Médale, J. L., Perrier, H., Romefort, D., Rouzaud, J., d'Uston, C., Möbius, E., Crocker, K., Granoff, M., Kistler, L. M., Popecki, M., Hovestadt, D., Klecker, B., Paschmann, G., Scholer, M., Carlson, C. W., Curtis, D. W., Lin, R. P., McFadden, J. P., Formisano, V., Amata, E., Bavassano-Cattaneo, M. B., Baldetti, P., Belluci, G., Bruno, R., Chioncho, G., Di Lellis, A., Shelley, E. G., Ghielmetti, A. G., Lennartsson, W., Korth, A., Rosenbauer, H., Lundin, R., Olsen, S., Parks, G. K., McCarthy, M., and Balsiger, H.: The Cluster Ion Spectrometry (CIS) Experiment, Space Science Reviews, 79, 303-350, 1997.

Rezeau, L., Roux, A., and Russell, C. T.: Can ULF fluctuations observed at the magnetopause play a role in anomalous diffusion?, Proceedings of the 26th ESLAB Symposium, Killarney, 127-131, 1992.

Rodriguez, P.: Magnetosheath electrostatic turbulence, J. Geophys. Res., 84, 917-930, 1979.

Rodriguez, P.: Magnetosheath whistler turbulence, J. Geophys. Res., 90, 6337-6342, 1985.

Samsonov, A. A.: Numerical modelling of the Earth's magnetosheath for different IMF orientations, Advances in Space Research, 38(8), 1652-1656, doi:10.1016/j.asr.2005.06.009, 2006.

Samsonov, A. A. and Hubert, D.: The steady-state slow shock inside the Earth's magnetosheath: to be or not to be? Part 2, Numerical modeling, J. Geophys. Res., 109, A01218, doi:10.1029/2003JA01006, 2004.

Sibeck, D. G., Lopez, R. E., and Roelof, E. C.: Solar wind control of the magnetopause shape, location and motion, J. Geophys. Res., 96, 5489-5495, 1991. 